\title{
Parteiendifferenz in der Umweltpolitik: Einleitung zum Themenheft
}

\author{
Simon Fink • Christiane Hubo • Annette Elisabeth Töller
}

Online publiziert: 11. Januar 2022

(C) Der/die Autor(en) 2022

\section{Einleitung}

Die Parteiendifferenztheorie ist ein Eckpfeiler der Policy Forschung. Sie geht davon aus, dass sich in westlichen Demokratien politische Parteien in ihren PolicyPositionen systematisch und grundsätzlich in vorhersagbarer Weise unterscheiden und dass sich diese Unterschiede auch in systematisch unterschiedlichen Policies niederschlagen, je nachdem, welche Parteien an der Regierung beteiligt sind.

Allerdings ist es umstritten, welche Erklärungskraft die Parteiendifferenztheorie für das Politikfeld Umwelt hat. Töller (in dieser Ausgabe) identifiziert drei grundlegende Probleme, die das Politikfeld Umwelt für die Parteiendifferenztheorie aufwirft: Das Problem der abhängigen Variablen, die Frage nach der singulären Kausalität und das Cleavage-Problem. Auch wenn alle Beiträge in diesem Themenheft ${ }^{1}$ diese drei grundlegenden Probleme implizit oder explizit adressieren, werden Lösungsansätze vor allem für das wichtigste Problem, das Cleavage-Problem vorgestellt.

\footnotetext{
1 Das Themenheft entstand auf der Grundlage des Workshops „Die Rolle politischer Parteien in der Umweltpolitik“ vom 07.-08.10.2020 in Göttingen, gefördert im Rahmen des DFG-Projektes „,Der Einfluss von Politiksektoren und Regierungswechseln auf Politikwandel im Waldnaturschutz“, Projektnummer 376182053, Abteilung für Forst- und Naturschutzpolitik, Georg-August-Universität Göttingen.
}

\footnotetext{
Simon Fink $(\bowtie)$

Institut für Politikwissenschaft, Georg-August-Universität Göttingen,

Platz der Göttinger Sieben 3, 37073 Göttingen, Deutschland

E-Mail: simon.fink@sowi.uni-goettingen.de

Christiane Hubo

Fakultät für Forstwissenschaften und Waldökologie, Abteilung für Forst- und Naturschutzpolitik, Georg-August-Universität Göttingen, Büsgenweg 3, 37077 Göttingen, Deutschland

Annette Elisabeth Töller

Lehrgebiet für Politikfeldanalyse und Umweltpolitik, FernUniversität in Hagen,

Universitätsstr. 33, 58084 Hagen, Deutschland
} 
Dieser einleitende Beitrag soll die grundlegenden Probleme kurz skizzieren und diskutieren, ob und wie die einzelnen Beiträge diese Probleme thematisieren oder sogar lösen. In der Zusammenschau fasst der einleitende Beitrag dann noch einmal generelle Lehren aus den einzelnen Beiträgen zusammen, zeigt Synergien sowie immer noch vorhandene Forschungslücken auf und skizziert eine zukünftige Forschungsagenda in diesem Feld.

\section{Problem 1: Dependent variable Problem}

Wendet man die Parteiendifferenztheorie auf die Umweltpolitik an, so besteht - wie in zahlreichen anderen Politikfeldern auch - das Problem der abhängigen Variablen. Auf welche Outcomes oder Outputs sollten sich Parteipräferenzen überhaupt auswirken? Und welche kausalen Mechanismen sind hier am Werk? Eine relativ kurze Kausalkette verbindet Parteipräferenzen mit Policies; länger, komplexer und vor allem sehr viel unsicherer wird die Kausalkette, wenn Umweltqualität oder Umweltperformanz die letztliche abhängige Variable sind (Töller in diesem Themenheft).

In diesem Themenheft findet sich erstens ein Beitrag, der Umweltqualität (also Outcomes) als abhängige Variable untersucht: der Beitrag von Detlef Jahn (in dieser Ausgabe) betrachtet die umweltpolitische Frage aus der Perspektive der makroquantitativen Staatstätigkeitsforschung. Im Gegensatz zu früheren Arbeiten, in denen Jahn Umweltperformanz als eine komplexe Variable erhob (Jahn 2016), betrachtet Jahn hier tatsächlich die Umweltqualität - in Form von Treibhausgasemissionen als abhängige Variable. In seiner international vergleichenden quantitativen Studie verwendet er die Stärke der Parteienfamilie an der Regierung (Anteil der Ministerien) als unabhängige Variable. Implizite Annahme über die Kausalkette zwischen Parteipräferenz und Umweltqualität ist also, dass Regierungsmehrheiten Policies beschließen, die sich dann auf die Umweltqualität auswirken. Um diesen Zusammenhang methodisch besser fassen zu können, verwendet der Beitrag von Jahn optimierte time lags. Er trifft also keine Annahmen darüber, wie lange es dauert, bis sich die Regierungsbeteiligung im letztlichen Outcome niederschlägt, sondern behandelt dieses Problem als empirische Frage.

Eine zweite Gruppe von Beiträgen behandelt Policies (i.e. den Output) als abhängige Variable. Hierzu gehört der Beitrag von Hubo und Göhrs, die auf Waldnaturschutzpolitik in den Bundesländern fokussieren und die Policies danach klassifizieren, ob sie eher dem Forst- oder dem Naturschutzsektor dienen. Der kausale Weg von den Parteipräferenzen zu den Policies ist relativ kurz, Hubo und Göhrs untersuchen sowohl die Regierungsbeteiligung als auch die Ressortverantwortung von Parteien (,Ministerial discretion“ (Laver und Shepsle 1998, S. 8 ff.)). Vor allem die Ressortverantwortung zeigt sich in der Empirie als wichtig. Auch der Beitrag von Schulze und Schönefeld hat eine Policy als abhängige Variable, in diesem Fall der Beitritt hessischer Kommunen zum Klimanetzwerk „Hessen aktiv: Die Klima-Kommunen“. Hier zeigt sich die Herausforderung bei der Anwendung der Parteiendifferenztheorie auf der kommunalen Ebene. Das kausale Argument bezieht folglich neben dem Bürgermeister die Gemeindevertretung mit ein: Die Parteifärbung des Bürgermeisters beeinflusst, ob eine Kommune dem Klimanetzwerk beitritt oder nicht. Das Ergreifen 


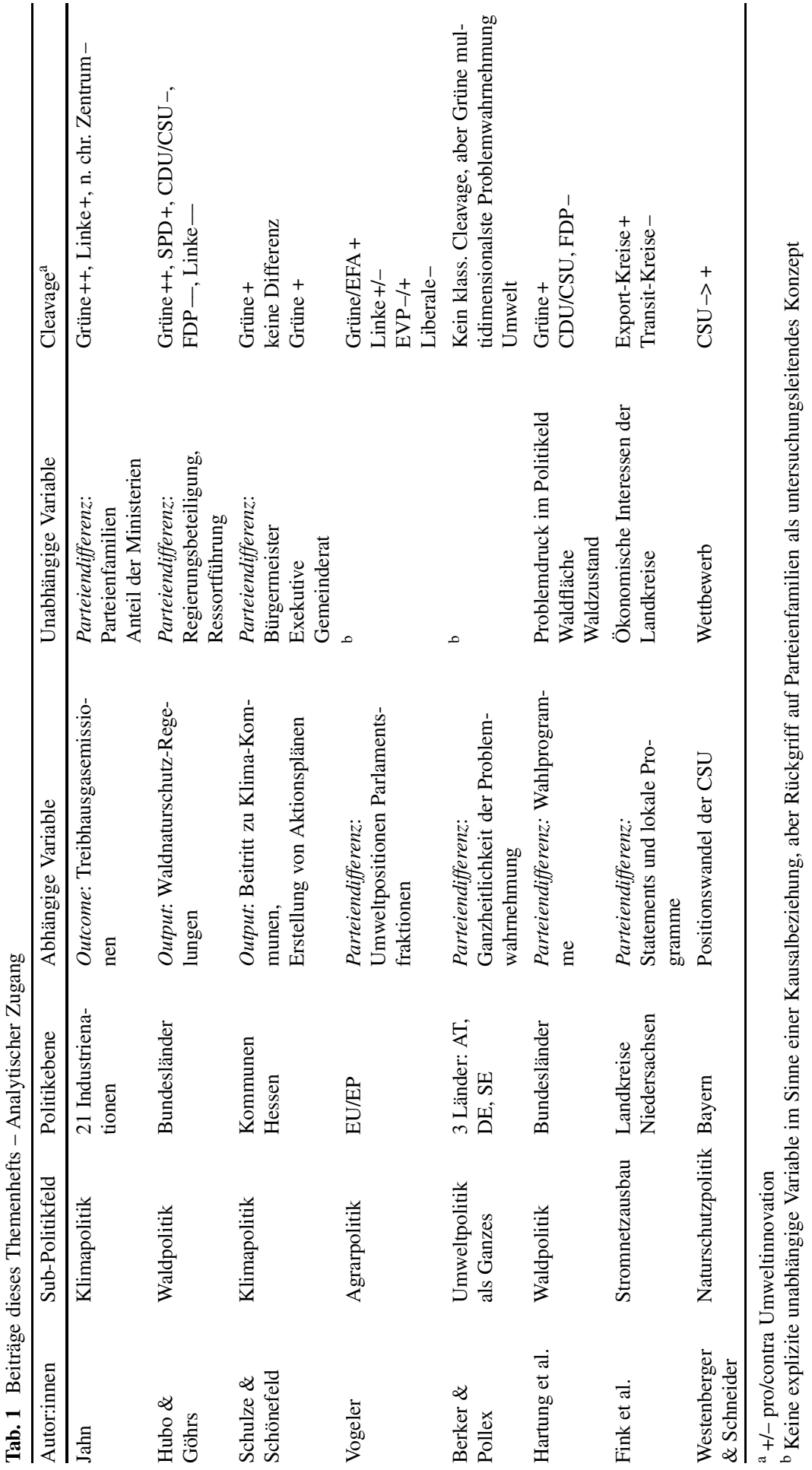


konkreter Schritte ist hingegen unabhängig von der Parteizugehörigkeit der lokalen Exekutive, variiert jedoch je nach Stärke der Grünen im Gemeinderat.

Die dritte und größte Gruppe von Beiträgen betrachtet weder Umweltpolitik (Output) noch Umweltqualität (Outcome) als abhängige Variable, sondern stellt die Parteienpositionen selber in den Mittelpunkt der Untersuchung (siehe Tab. 1).

Damit decken die Beiträge eine interessante Bandbreite möglicher abhängiger Variablen ab, ohne dass allerdings die von Töller aufgeworfene Problematik gelöst würde: Die angenommenen Kausalketten sind entweder relativ kurz und verbinden Regierungen bzw. Regierungsmehrheiten mit Policies. Sind die Kausalketten aber lang und reichen von der Parteiendifferenz bis zur Umweltqualität, bleiben einige Teile der Kausalkette implizit.

\section{Problem 2: Singuläre Kausalität}

Das zweite von Töller (in dieser Ausgabe) identifizierte Problem ist die Frage der Kausalität: welchen Einfluss haben Parteien auf umweltpolitischen Output oder Outcome, wenn zahlreiche andere Faktoren - Institutionen oder sozioökonomischer Kontext - sich ebenso auf Policies oder Umweltqualität auswirken? Die Rolle von, wenn man so will, intervenierenden Faktoren können zwangsläufig nur diejenigen Studien adressieren, die klassischerweise einen kausalen Zusammenhang zwischen Output oder Outcome und Parteipolitik zu etablieren versuchen.

Jahn beantwortet diese Frage - der Logik der quantitativen Herangehensweise folgend - mit dem Hinzufügen eines Korporatismus-Indikators als Kontrollvariable. Da dieser Beitrag mit den Treibhausgasemissionen auch die längste Kausalkette aufspannt, sind außerdem Variablen wie Anteil fossiler Brennstoffe und Wirtschaftswachstum in den Modellen enthalten. Der Beitrag folgt also dem Argument, den relativen Einfluss von Parteienideologie zu ermitteln, der übrig bleibt, wenn man für große sozioökonomische Trends kontrolliert.

Die Beiträge von Hubo und Göhrs sowie Schulze und Schönefeld ziehen eine relativ klare Kausalkette von der Besetzung der Exekutive durch Parteien zu Policies. Schulze und Schönefeld fügen ihren Regressionsmodellen eine Reihe von Kontrollvariablen hinzu: Bevölkerungsdichte, Beschäftigte in der Industrie, Steuereinnahmen und Verschuldung sollen - ähnlich wie bei Jahn - dafür kontrollieren, dass Kommunen höchst unterschiedlich sind und die Parteipräferenz des Bürgermeisters/der Bürgermeisterin nicht die einzige Variable ist, die Umweltpolicies beeinflusst.

Damit adressieren alle drei Beiträge, die auf eine Kausalerklärung abzielen, die Bedingtheit des Einflusses von Parteipolitik, wenn auch auf eher konventionelle Weise.

\section{Problem 3: Cleavage-Struktur}

Das größte Problem bei der Anwendung der Parteiendifferenztheorie auf die Umweltpolitik besteht in den Fragen, ob es eine herausstechende Konfliktlinie im Politikfeld gibt und wie diese ggf. im Parteiensystem abgebildet ist. 
Töller argumentiert in ihrem Beitrag, dass es zwar in der Umweltpolitik unterschiedliche Problemstrukturen mit unterschiedlichen Konfliktlinien gebe, dass aber in der Mehrzahl der Fälle die Konfliktlinie Ökologie versus Ökonomie prägend sei. Sie führt weiter aus, dass die Parteiendifferenztheorie mit unterschiedlicher theoretischer Unterfütterung angewendet wird, was zu unterschiedlichen Annahmen hinsichtlich der Abbildung dieser Konfliktlinie im Parteienspektrum führt. Töller unterscheidet drei verschiedene Ansätze:

Die ,alte Parteientheorie“ (die Unterscheidung in alte und neue Parteientheorie machen Wenzelburger und Zohlnhöfer 2020), wie sie z. B. von Hibbs (1977) vertreten wurde, ging traditionell davon aus, dass bestimmte Wählerinteressen stabil von bestimmten Parteien vertreten werden und dass die Unterscheidung zwischen rechten und linken Parteien für die Policypositionen (und die tatsächlich verfolgte Politik) entscheidend sei. Das Aufkommen der ökologischen Konfliktlinien sowie der Partei der Grünen integrierte Kitschelt in diese Rechts-Links-Achse, indem er argumentiert, die Modernisierung habe zu einer Verschiebung der Hauptachse der Wählerverteilung und der Parteienkonkurrenz von einer einfachen Rechts-links- (oder kapitalistisch vs. sozialistisch) Konfliktlinie zu einer neuen, komplexeren, „quer“ liegenden Konfliktlinie geführt (Kitschelt 1994). Demnach wären ökologische Positionen eher bei Parteien auf der links-libertären Seite des Kontinuums (also von Grünen und auch von linken Parteien) zu erwarten.

In Abgrenzung hierzu argumentieren Vertreter:innen der „nеиеn Parteientheorie ", dass Parteien auf die Veränderung von bestehenden Konfliktlinien und das Hinzukommen neuer Konfliktlinien in der Wählerschaft reagierten, indem sie - motiviert vom Interesse am Erhalt elektoraler Unterstützung - maßgeschneiderte Angebote für bestimmte Wählergruppen machten (z. B. Gingrich und Häusermann 2015). Parteien verfolgten in einem Bereich so lange unterschiedliche Policies, wie es unterschiedliche Präferenzen bei den Wähler:innen gebe und unterschiedliche Policypositionen eine Wiederwahl wahrscheinlicher machten (Wenzelburger und Zohlnhöfer 2020). Diese Überlegung zur Absorption neuer Konfliktlinien in Parteiideologien würde nahelegen, dass Parteien ökologische Interessen dann aufgreifen, wenn sie von ihrer Wählerschaft nachgefragt werden. Solange also Wähler:innen mit ökologischen Interessen vorrangig grüne und oder linke Parteien wählen, würde dies zu einem ökologischen Profil in Programmatik und Politik von grünen oder linken Parteien führen. Es könnte jedoch auch zu einer Verbreitung von ökologischen Positionen über das (beinahe) gesamte Parteienspektrum (,greening the mainstream“, Carter 2013) kommen.

Die Theorie der Parteienfamilien (von Beyme 2000, S. 70 f.) betont die historische Entstehung und die ideologische Substanz, die bestimmte Parteien als Parteienfamilien verbindet. Wenn für die Verarbeitung neuer Konfliktlinien in den Parteien nicht die elektorale Nutzenerwartung, sondern ihre historisch geprägte Identität entscheidend ist, dann wäre in erster Linie von grünen Parteien zu erwarten, dass sie ökologische Fragen in ihren Programmen prioritär adressieren und an der Regierung auch tatsächlich restriktive Umweltpolitiken verfolgen. Für linke Parteien sollten hingegen Fragen der sozialen Gerechtigkeit und der Umverteilung im Mittelpunkt stehen, so dass eine Absorption ökologischer Positionen eher an der Oberfläche stattfände. 
Während in den makro-quantitativen Studien zur Parteiendifferenztheorie diese verschiedenen theoretischen Unterfütterungen genutzt wurden, um plausible Annahmen über Parteienpositionen zu umweltpolitischen Fragen gewissermaßen ,,a priori“, also ohne Blick auf die tatsächliche Programmatik, zu treffen, können diese ebenso gut eine empirische Herangehensweise anleiten, die an die Stelle von a-priori-Annahmen zu den Parteienpositionen empirische Erhebungen stellt, also schaut, welche Positionen die Parteien zu Umweltfragen denn tatsächlich haben.

Diese unterschiedlichen Herangehensweisen finden sich auch in den Beiträgen des Themenhefts wieder. Es gibt Beiträge mit a-priori-Annahmen und solche, die die Parteienpositionen empirisch erheben. Die meisten Beiträge nehmen implizit oder explizit Bezug zur Parteienfamilientheorie, aber es gibt auch Bezüge zu den Annahmen der alten und der neuen Parteientheorie.

Die erste Gruppe von Beiträgen adressiert das Problem im Rahmen üblicher, auf Kausalerklärung ausgerichteter Forschungsdesigns, wobei die Annahmen über Parteienpositionen letztlich a-priori aus den entsprechenden Theorien (Parteienfamilienansatz, Parteientheorien) abgeleitet werden: Der Beitrag von Jahn geht von einer Einteilung der Parteien in historisch entwickelte Parteienfamilien aus, ebenso der Beitrag von Schulze und Schönefeld und implizit auch der von Hubo und Göhrs. Interessant ist vor allem die Frage, welche Annahmen zu linken (aber nicht grünen) Parteien getroffen werden und ob sich diese bestätigen. Jahn zeigt, dass sowohl grüne als auch linke Regierungsbeteiligung mit der Senkung von Treibhausgasemissionen korreliert, und Hubo und Göhrs sehen einen Wandel zu Waldnaturschutz durch grüne und sozialdemokratische Ressortverantwortung, jedoch nicht durch die Partei Die Linke. Schulze und Schönefeld sehen den Unterschied dagegen vor allem zwischen den Grünen und dem Rest des Parteiensystems, nur grüne Bürgermeister:innen machen den Beitritt zum Klimanetzwerk der hessischen Kommunen wahrscheinlicher. Damit decken unsere Beiträge auch empirisch das Spektrum, das sonst in der Literatur vertreten wird (siehe auch Potrafke 2017), sehr gut ab.

Vor dem Hintergrund, dass a-priori-Annahmen zu parteipolitischen Positionen in der Umweltpolitik jenseits der Positionen der Grünen problematisch erscheinen, untersucht eine zweite Gruppe von Beiträgen (Vogeler; Berker und Pollex) empirisch, welche parteipolitischen Muster sich im Hinblick auf eine bestimmte Policy-Frage ergeben. Der Beitrag von Vogeler untersucht anhand einer Diskursnetzwerkanalyse, wie sich die politischen Gruppen im Europäischen Parlament (EP) zur Agrarpolitik positionieren. Die Analyse zeigt einerseits eine klare Parteiendifferenz zwischen den grünen und den konservativen politischen Gruppen im EP; andererseits aber auch einen parteienübergreifenden Konsens, dass Nachhaltigkeit für die Agrarpolitik wichtig ist. Der Beitrag von Pollex und Berker schlägt vor, die Positionen von Parteien in Deutschland, Österreich und Schweden zu Umweltthemen darüber zu konzeptualisieren, wie ganzheitlich ihre Problemwahrnehmung und -definition von Umweltpolitik sind. Darunter verstehen sie die Frage, welche Dimensionen von Umweltproblemen Parteien in ihren Programmen ansprechen: Ihr Gemeinwohlcharakter, ihre Persistenz, ihre räumliche und zeitliche Dimension, die damit verbundene Unsicherheit, die Integration von Umweltfragen in andere Politikfelder und die soziale Dimension. Auf diese Weise können sie zeigen, dass Parteien sich nicht nur in ihren Positionen zu Umweltpolitik unterscheiden, sondern auch danach, wie ein- oder 
mehrdimensional sie diese Umweltpolitik konzeptualisieren und sie z. B. auch die sozialen Fragen, die Umweltpolitik aufwirft, in ihr Konzept von Umweltproblemen mit aufnehmen. Im Ergebnis unterstützt der Beitrag von Pollex und Berker eher das Argument der Grünen als umweltpolitischem Spezialfall. In ihrem Beitrag heben sich die Grünen vor allem durch ihre holistische Problemwahrnehmung von allen anderen Parteien ab. Implizit gehen beide Beiträge vom Konzept der Parteienfamilien aus. Zwar werden keine a-priori-Annahmen über Parteienpräferenzen getroffen, aber dennoch bauen die Hypothesen über die erwarteten empirischen Regelmäßigkeiten darauf auf, dass Parteien historische Markenkerne haben und deshalb z. B. die Abgeordneten der Europäischen Volkspartei nah an den Interessen der Landwirtschaft liegen (Vogeler) oder grüne Parteien im europaweiten Vergleich einen gemeinsamen Markenkern haben (Berker und Pollex).

Eine dritte Gruppe von Beiträgen kombiniert eine solche empirische Untersuchung mit dem Anliegen, diese parteipolitischen Muster wiederum zu erklären. In dieser letzten Gruppe sind die parteipolitischen Positionen und Muster abhängige und nicht unabhängige Variable (siehe Tab. 1). Hartung et al. sehen die Konfliktlinie in der Waldpolitik zwischen Grünen auf der einen und FDP/CDU auf der anderen Seite, für die Linke, die SPD und die AfD sei das Politikfeld dagegen in den Parteiprogrammen nur wenig salient. Sie argumentieren, dass die Salienz der Waldpolitik davon abhängt, wie viel Waldanteil ein Bundesland hat, ob es ein ostoder westdeutsches Bundesland ist, und wie groß der Anteil naturnahen Waldes ist. Parteipositionen werden also nicht nur auf die Mitgliedschaft in einer Parteienfamilie zurückgeführt - wobei der Beitrag die klassischen Parteienfamilien als Variablen verwendet - sondern es wird angenommen, dass Parteienpositionen wiederum durch regionale Kontextfaktoren bestimmt sein könnten. Somit kombiniert der Beitrag Annahmen der Parteienfamilien-Sichtweise mit der neuen Parteientheorie. Noch stärker in der neuen Parteientheorie verortet sind Fink et al., die zeigen, dass es für die Positionen niedersächsischer kommunaler Parteien zu Stromnetzausbau vor allem darauf ankommt, ob der Landkreis vom Stromnetzausbau profitiert oder nicht. Die ökonomischen Interessen des Landkreises überlagern die Präferenzen, die man auf der Basis der Parteienfamilien eigentlich erwarten würde. Die Studie von Westenberger und Schneider argumentiert, dass der Positionswandel der CSU sich vor allem aus dem Wettbewerb um Wählerstimmen erklären lässt - wenn die öffentliche Meinung pro Umweltschutz ist und die Wähler:innen die umweltpolitischen Positionen anderer Parteien honorieren, zieht die CSU nach. Im Ergebnis ist der Befund sowohl von Westenberger und Schneider als auch von Fink et al., dass es eher keine Parteiendifferenz gibt. In Bayern hat sich die CSU zumindest rhetorisch sehr stark den Grünen angenähert und den nord- und südniedersächsischen Landkreisen überwiegen ökonomisch determinierte Unterschiede zwischen Parteien. Dies unterstützt interessanterweise die Annahmen der neuen Parteientheorie, die davon ausgeht, dass sich Parteien relativ flexibel an den Policy-Präferenzen ihrer Wähler:innen ausrichten.

Für den empirischen Zugriff stellt sich die Frage, wie die Parteiendifferenzen operationalisiert und gemessen werden. Hier bietet das Themenheft eine Vielfalt an Ansätzen. Beispielsweise ermitteln Hartung et al. und Fink et al. die Parteienpositionen anhand von Wahlprogrammen und Statements. Der Beitrag von Vogeler 
verwendet Aussagen von Abgeordneten des EU-Parlaments in Parlamentsdebatten, um in einer Diskursnetzwerkanalyse herauszufinden, inwieweit Umweltpositionen in den agrarpolitischen Positionen der Parteien auf EU-Ebene aufgenommen wurden. Auf der Seite der a-priori-Ansätze verfolgen Schulze und Schönefeld in ihrem Beitrag den Ansatz, die Parteizugehörigkeit der Bürgermeister:innen mit dem Beitritt zu den hessischen Klima-Kommunen, Hubo und Göhrs korrelieren die Regierungsbeteiligung und die Ressortzuständigkeit der Landesparteien mit der politischen Ausrichtung von Waldnaturschutzregelungen und Jahn verwendet ganz klassisch den Anteil der Parteien an den Ministerien zur Messung der Stärke von Parteienfamilien. Der Beitrag von Westenberger und Schneider fokussiert hingegen auf den Ministerpräsidenten, um die Aufnahme von Umweltpositionen der CSU im Kontext des Parteienwettbewerbs darzustellen.

Die Beiträge umfassen damit auch eine große Bandbreite hinsichtlich der territorialen Ebenen: sie reicht von der kommunalen Ebene bei Fink et al. und Schulze und Schönefeld über die Bundesländer bei Hubo und Göhrs, Hartung et al. sowie Westenberger und Schneider bis zu internationalen Vergleichen bei Berker und Pollex sowie Jahn.

Weiterhin befassen sich die Beiträge mit Konfliktlinien in unterschiedlichen Subfeldern der Umweltpolitik, insbesondere mit Naturschutzkonflikten in der Landund Forstwirtschaft sowie klima- und energiepolitischen Konflikten. Lassen sich über diese unterschiedlichen Ebenen und Felder hinweg Gemeinsamkeiten in den Aussagen zur Parteiendifferenz feststellen? Sehr deutlich ist, dass in allen Beiträgen die grünen Parteien den stärksten Einsatz für die Umweltpolitik zeigen, und zwar sowohl bei den Parteienpositionen als auch im Regierungshandeln. Am gegenüberliegenden Pol rangieren liberale Parteien, ebenfalls bei den Parteienpositionen und den von ihnen verantworteten Policies, zumindest in Deutschland und der EU. Bei allen anderen Parteien sind die Ergebnisse uneinheitlich. Für konservative Parteien (CDU/CSU, EVP) kann gesagt werden, dass sie sich in den Naturschutzkonflikten mit der Land- und Forstwirtschaft eher zugunsten von Landnutzungen positionieren und dies auch im Regierungshandeln umsetzen. Etwas heterogener sind die Ergebnisse für linksorientierte Parteien: bei den Treibhausgasemissionen der 21 Industrienationen und der EU-Agrarpolitik sind sie eher pro Umweltschutz zu verorten; in der Waldnaturschutzpolitik der deutschen Bundesländer thematisieren linke Parteien das Thema in den Wahlprogrammen kaum, doch im Regierungshandeln ist die SPD eher pro, die Linke hingegen kontra Umweltpolitik ausgerichtet.

In der Summe bringen die Beiträge dieses Themenhefts etwas mehr Klarheit in das relativ heterogene Bild der umweltpolitischen Cleavages, das in der Literatur herrscht.

\section{Zusammenfassung und Ausblick}

Sowohl das Problem der abhängigen Variablen als auch das Problem der singulären Kausalität werden in den Beiträgen unseres Themenheftes durchaus adressiert, jedoch nicht gelöst. 
Hingegen widmen sich alle Beiträge - wenn auch auf recht unterschiedliche Weise - dem wichtigsten Problem, dem Cleavage-Problem. Mit jeweiligen ihrem Vorgehen löst die Mehrzahl der Beiträge einerseits das beschriebene Problem, dass Positionen gerade in der Umweltpolitik nicht ohne Weiteres a-priori unterstellt werden können.

Dabei beschränken sich einige Studien darauf, die Parteipositionen zu erheben, während andere zudem der Frage nach den Ursachen der Parteipositionen nachgehen. In einer solchen Fokussierung auf die empirische Erhebung der Parteipositionen (deren Umsetzung in Policies überprüft wird oder auch nicht) entsteht jedoch, was Reimut Zohlnhöfer als ,,independent variable Problem“ bezeichnet hat: Es verschiebt sich die Forschungsfrage ,from asking whether parties make a difference in public policy to the question whether parties in government do what they promise in their manifestos“ (Zohlnhöfer 2020, S. 3). Die ursprüngliche Frage der Parteiendifferenzforschung, ob es einen systematischen Unterschied macht, welche Partei an der Regierung ist, kann so jedoch nicht beantwortet werden (ebd., S. 4-6). Allerdings deuten die Ergebnisse der Beiträge in der Gesamtschau darauf hin, dass es bei den meisten Parteien (grünen, liberalen, auch konservativen) durchaus diesen systematischen Unterschied gibt, der auch gut erklärt werden kann. Die Forschungsfrage wird dabei in den vorliegenden Beiträgen um die nach der Einlösung von Wahlversprechen teilweise ergänzt, jedoch von dieser sicher nicht abgelöst. Fraglich ist jedoch, inwieweit die vorliegenden empirisch und theoretisch fundierten Ergebnisse bereits eine ausreichende Grundlage für verallgemeinerungsfähige Aussagen bilden. Weitere Untersuchungen sind hier angezeigt.

Letztlich muss die Herangehensweise des „Nur“-empirischen Messens nicht der Weisheit letzter Schluss sein. Wie Töller in ihrem Beitrag argumentiert, sollten auf der einen Seite die gemessenen Positionen in der zukünftigen Forschung verstärkt in Relation zu Policy Outputs (und ggf. auch Outcomes) gesetzt werden. Darüber hinaus wäre das Zohlnhöfer-Argument zu entkräften, indem man auf der anderen Seite die gemessenen Policy-Positionen systematisch in Relation zu den (verschiedenen) a-priori-Annahmen der Parteiendifferenztheorie setzt - oder, wie hier bereits ansatzweise geschehen, welche Faktoren anstelle der Tradition (Parteienfamilien) oder des Wahlkalküls (Parteientheorien) die Parteipositionen beeinflussen. Damit deutet sich auch die Forschungsagenda an, die dieses Themenheft umreißt. Besonderen Forschungsbedarf werfen zudem die heterogenen Ergebnisse zu den linken Parteien auf. Hier wäre in der zukünftigen Forschung zu untersuchen, ob die Unterschiede in den Parteipositionen durch bestimmte zusätzliche Bedingungen erklärbar sind.

Funding Open Access funding enabled and organized by Projekt DEAL.

Open Access Dieser Artikel wird unter der Creative Commons Namensnennung 4.0 International Lizenz veröffentlicht, welche die Nutzung, Vervielfältigung, Bearbeitung, Verbreitung und Wiedergabe in jeglichem Medium und Format erlaubt, sofern Sie den/die ursprünglichen Autor(en) und die Quelle ordnungsgemäß nennen, einen Link zur Creative Commons Lizenz beifügen und angeben, ob Änderungen vorgenommen wurden.

Die in diesem Artikel enthaltenen Bilder und sonstiges Drittmaterial unterliegen ebenfalls der genannten Creative Commons Lizenz, sofern sich aus der Abbildungslegende nichts anderes ergibt. Sofern das betreffende Material nicht unter der genannten Creative Commons Lizenz steht und die betreffende Handlung 
nicht nach gesetzlichen Vorschriften erlaubt ist, ist für die oben aufgeführten Weiterverwendungen des Materials die Einwilligung des jeweiligen Rechteinhabers einzuholen.

Weitere Details zur Lizenz entnehmen Sie bitte der Lizenzinformation auf http://creativecommons.org/ licenses/by/4.0/deed.de.

\section{Literatur}

von Beyme, Klaus. 2000. Parteien im Wandel: von den Volksparteien zu den professionalisierten Wählerparteien, 1. Aufl., Wiesbaden: Westdeutscher Verlag.

Carter, Neil. 2013. Greening the mainstream: party politics and the environment. Environmental Politics 22:73-94.

Gingrich, Jane, und Silja Häusermann. 2015. The decline of the working-class vote, the reconfiguration of the welfare support coalition and consequences for the welfare state. Journal of European Social Policy 25:50-75.

Hibbs, Douglas A. 1977. Political parties and macroeconomic policy. American Political Science Review 71:1467-1487.

Jahn, Detlef. 2016. The politics of environmental performance. Cambridge: Cambridge University Press.

Kitschelt, Herbert. 1994. The transformation of european social democracy. Cambridge: Cambridge University Press.

Laver, Michael, und Kenneth A. Shepsle. 1998. Cabinet ministers and government formation in parliamentary democracies. In Cabinet ministers and parliamentary government, Hrsg. Michael Laver, Kenneth A. Shepsle, 3-12. Cambridge: Cambridge University Press.

Potrafke, Niklas. 2017. Partisan politics: the empirical evidence from OECD panel studies. Journal of Comparative Economics 45:712-750.

Wenzelburger, Georg, und Reimut Zohlnhöfer. 2020. Bringing agency back into the study of partisan politics: a note on recent developments in the literature on party politics. Party Politics 27:1055-1065.

Zohlnhöfer, Reimut. 2020. The analysis of partisan differences in advanced democracies. The "independent variable problem" reconsidered. Unveröffentlichtes Manuskript. 